\title{
Das Ende des Heidentums in Hispania
}

\author{
Rosa María Sanz Serrano
}

Universidad Complutense de Madrid

ZuSAMMENFASSUnG: Die Quellen zur spätantiken Religionsgeschichte der Iberischen Halbinsel werden durch eine christliche determinierte Voreingenommenheit dominiert. Die Tendenz der Quellen geht dahin, die Existenz des Heidentum im 5., 6. und 7. Jahrhundert zu bestreiten: Stattdessen wird behauptet, alle Einwohner seien Christen und religiöse Konflikte ergäben sich lediglich aufgrund von Aberglauben, magischen Praktiken oder christlichen Häresien. Dieser Aufsatz stellt die These auf, dass das Heidentum sehr viel länger mit dem Christentum koexistiert hat, als allgemein angenommen wird.

Schlagworte: Hispania, Religionskonflikte, Heidentum, Christentum, Spätantike.

AbSTRACt: Sources about the religious history of the Iberian Peninsula in Late Antiquity are dominated by a Christian bias. The general tendency of those sources is to negate the very existence of Paganism in the $5^{\text {th }}, 6^{\text {th }}$, and $7^{\text {th }}$ century: Instead, it is claimed that all inhabitants are Christians and religious conflicts are explained as arising from superstition, magical practices or Christian heresies. This article argues that Paganism coexisted with Christianity much longer than is commonly assumed.

Keywords: Hispania, religious conflict, paganism, Christianity, Late Antiquty. 
$\mathrm{W}$ ollen wir den Wandel der religiösen Vorstellungen in Hispania analysieren, müssen wir uns darüber klar werden, was wir am Ende des Altertums als Paganismus oder Heidentum betrachten können. Dabei handelt es sich eigentlich um eine erdachte Kategorie, in der unterschiedliche Mythen, Glaubensrichtungen, Riten und andere Gebräuche durch das Christentum zusammengefasst und vereinheitlicht wurden. Entscheidend zum Verständnis des Paganismus ist deshalb die Frage, wie er sich in die Romanitas Christiana wandelte. Diese Umwandlung allein verhindert oft schon eine historische Objektivität. Ferner spielt die Zerstörung heidnischer Dokumente in privaten und öffentlichen Räumen durch Kirche und Staat eine wichtige Rolle. Diese Zerstörung wird nicht nur durch entsprechende Gesetze bezeugt, sondern in besonderen Fällen auch durch die Vernichtung heidnischer Bücher und die Übergabe der staatlichen Bildung in die Hände von Bistümern und Klöstern. Ohne diese Übergabe an kirchliche Institutionen wäre es zum Beispiel nicht möglich gewesen, die Etymologiae des Isidor von Sevilla zu erarbeiten. Alle diese Aktionen hatten das Ziel, heidnische Propaganda zu verhindern und ferner ein Bild zu entwerfen, nach dem der Paganismus ein Zeichen kultureller Armut sei, schriftlos und unfähig, mit der Kultur des christlichen Glaubens zu konkurrieren, gleichsam der Gegenpol zu einer Welt, in der der ideologische Diskurs des Christentums das Idealmodell der spätantiken Gesellschaft gestalten konnte. Die kulturelle, politische und geschichtliche Hegemonie des Christentums sollte damit definitiv bestätigt werden. Dieses Monopol wurde von einer neuen Art der Beziehung zwischen dem Staat und seinen Untertanen unterstützt, wonach zum Beispiel unter Androhung empfindlicher Strafen bestimmte Kulte verboten und Tempel geschlossen wurden. ${ }^{1}$

Die Art und Weise, wie die Geschichte des Christentums erzählt wird, beeinflusst deshalb auch unser gegenwärtiges Konzept vom Ende des Paganismus, der auf der Iberischen Halbinsel durch eine Vielzahl von Kulten repräsentiert wurde, deren individuelle Züge häufig nicht berücksichtigt werden. So werden der Kult des Iupiter Capitolinus oder des Melkart in Gades oder des lusitanischen Endovellicus gleichartig behandelt. Die Gewalt der Eroberung wird dabei durch die der ideologisch-religiösen Kontrolle ersetzt. Damit wird der Heide in Randmilieus verdrängt, wie sie Orosius (Hist.VII, 9) sehr gut beschrieben hat, indem er von zurückgebliebenen Bauern an

1. Zu diesem Wandel: K. Hopkins: A World full of Gods. Pagans, Jews and Christians in the Roman Empire, London, 1999; G. CAvallo: «L'altra lettura. Tra nuovi libri e nuovi testi», Ant. Tard. 9 (2001), 131 138. Außerdem: P. Brown: Power and Persuasion in Late Antiquity, Wisconsin 1992; W. V. HARRIS: Ancient Literacy, Harvard 1989; H. Leppin: Der Prediger und der Mönch. Zur Bewertung christlicher Rede in der Spätantike, in: C. NeUmeISTER-W. RAEK (Hg.): Rede und Redner. Bewertung und Darstellung in den antiken Kulturen, Möhnesee, 2000, 301-312; C. Markschies: «Lehrer, Schüler, Schule: Zur Bedeutung einer Institution für das antike Christentum», in: U. EgelhaAf-GAiser, A. SCHÄFer (Hg.): Religiöse Vereine in der römischen Antike, Tübingen, 2002, 97-119 und R. Lim: Public Disputatio. Power and Social Order in Late Antiquity, Berkeley, 1995; C. Bertelli: «The production and distribution of books in Late Antiquity», in: R. Hodges,W. Boden (Hg.): The sixth century. Production, Distribution and Demand, Leiden, 1998, 41-60; S. LANCEL: Saint Augustin, Paris, 1999. Gute Gedanken dazu R. A. KASter: Guardians of language: The Grammarian and Society in Late Antiquity, California, 1988. 
entlegenen ländlichen Orten spricht: locorum agrestium conpitis et pagis. Dies ähnelt den gentiles qui terrena sapiunt, die sich also auf irdische Dinge konzentrieren. Dieses Modell wiederholt sich ab dem 6. Jahrhundert in der De correctione rusticorum des Martin von Braga, bei Isidor von Sevilla (Etym. VIII, 9, 1-4) sowie auf den Konzilien, wo man den Heiden zutraut, unglaubliche Taten zu begehen: multaque alta quae per longum est enarrare (XVI. Konzil von Toledo [693], can. 2). Damit suchte man den Gegensatz zur klassischen Kultur zu betonen und insbesondere die Gegensätze Stadt - Land, Indigene Romanisierte, Zivilisation - Barbarei herausstellen. ${ }^{2}$

Bei anderen Gelegenheiten ist von Apostasie, Idolatrie oder Aberglauben die Rede, wobei gerade in den Werken von Hydatius und Orosius vieles durcheinander gerät, und zwar als Ergebnis von Ablehnung und Widerwillen gegenüber nicht akzeptierten Glaubensrichtungen. Anschuldigungen gegen christliche Abweichler wurden auf den Konzilien als Bräuche der Heiden dargestellt (paganorum consuetudinem, traedictiones gentilium, diabolica suprestitiones errores), wobei es sich natürlich um Dinge handelte, die im Gegensatz zu den propagierten Konzepten vom christlichen Abendland standen. ${ }^{3}$ Parallel dazu kommt es zu einer Verspottung heidnischer Götter, vor allem in der Literatur werden sie lächerlich gemacht. Die Dämonisierung ist dabeieinweitereskünstlichesundliterarisches KonstruktderGegenüberstellung von Gut und Böse im Sinne von heidnischen Göttern und ihrem Gegenüber, den christlichen Heiligen. Erstere leiten den Flug der Zugvögel, bewegen Statuen, ordnen Träume und Prophezeiungen, bringen aber auch Krankheiten und Unglück, nachdem sie aus dem Himmel vertrieben wurden. Auf dem 16. Konzil von Toledo (can. 2) verwandeln sie sich schließlich in den Feind des Menschengeschlechts (hostis humani generis), der um die Welt tobt und auf der Suche ist, jemanden zu verschlingen: per mundum rugiens currat quaerens quem devoret. ${ }^{4}$

2. Prudentius, contra Symm. I, 435-50; ep. 31,203; Eutropios ep. PL Suppl. I, 555. Ich habe dieses Problem behandelt in: «Paganos, adivinos y magos. Análisis del cambio religioso en la Hispania Tardoantigua», Anejos Gerión, Nr. 7, 2003, 40f.

3. Zum Beispiel der Kanon 46 des Konzils von Elvira, welches Apostasie mit Idolatrie gleichsetzt. Auch Martin von Braga (De correct.,7) widmete im Jahr 572 diesem Thema sein Werk: Pro castigatione rusticorum, qui adhuc pristina paganorum superstitione de gentes cultum venerationis plus daemoniis quam deo persolvunt[...]. In Westgotischen Zeiten beim III. Konzil von Toledo, can. 16, XII. Konzil von Toledo, can. 11 und XVI. Konzil von Toledo, can. 2., zitiert nach: J. VIVEs: Concilios visigóticos e hispanorromanos, Barcelona-Madrid, 1963. Außerdem: R. M. Sanz Serrano: «Paganos, adivinos y magos: análisis del cambio religioso en la Hispania Tardoantigua», 97f. und das alte Werk von S. McKennA: Paganism and Pagan Survivals in Spain up to the Fall of Visigothic Kingdom, Washington, 1938. Für Gallien siehe N. ZEDDIES, Religio et sacrilegium. Studien zur Inkriminierung von Magie, Häresie und Heidentum (4.-7. Jahrhundert), Bern, 2003.

4. Augustinus, doctr. christ.., II; Orosius, Hist.VII, 29, 2; Dumiensis, 16: Ecce qualis cautio et confessio vestra apud deum tenetur! Et quomodo aliqui ex vobis, qui abrenuntiaverunt diabolo et angelis eius et culturis eius et operibus eius malis, modo iterum ad culturas diaboli revertuntus? und XII. Konzil von Toledo, can. 11. Siehe R. M. Sanz Serrano: «Santos y demonios como elementos de cristianización en Occidente», in: J. Alvar, C. Blánquez, C. G. Wagner (Hg.): Héroes, semidioses y Daimones, Madrid, 1992, 463-484. Dort erörtere ich einige der Theorien von P. Brown: Society and the Holy in the Late Antiquity, London, 1982 und DERS.: The cult of the saints. Its rise and function in Latin Christianity, Chicago, 1981. 
Im Grunde aber sind unsere Informationen wenig verlässlich. Im 4. Jahrhundert, als zweifellos noch eine heidnische Mehrheit existierte, berichten die Quellen größtenteils nur von organisatorischen Aspekten der hispanischen Kirche, ausgenommen einiger Kanons des Konzils von Elvira. Sogar die von Priscillian angeführte Bewegung wird als häretisch dargestellt, geprägt von gnostischen Lehren und durchaus in der Lage, die noch unsichere kirchliche Struktur zu gefährden. Diese Auseinandersetzung wird dabei als Streit zwischen südlichen Bischöfen unter Führung von Hydatius von Emerita und Itacius von Ossonoba und den Priscillianern gesehen, welche die wichtigsten Bischofssitze des Nordens kontrollierten, an die sich das Reskript Gratians aus dem Jahre 381 richtete (Cod. Theod. XVI, 2, 35). ${ }^{5}$ Auf diese Weise wollten die Quellen verbergen, dass es in der Bewegung des Priscillian viele heidnische Anhänger gab, die beschuldigt wurden, Sonne und Mond, Tiere sowie jüdische Götter und Götzen zu verehren, was von mehreren Kanons der in Braga im 6. Jahrhundert einberufenen Konzilien festgestellt wurde, um sie zu exkommunizieren. Doch die christlichen Quellen weigern sich weiterhin, den heidnischen Charakter der Priscillianer anzuerkennen, obwohl diese dafür verurteilt wurden. Dies ist zu erwarten bei einer von Adeligen angeführten Bewegung, welche laut Sulpicius Severus viele heidnische Abhängige mitriss (multos nobilium pluresque populares). ${ }^{6}$ Der politische Prozess, der ihrer Verurteilung folgte, hing mit dem Weiterleben heidnischer Glaubensvorstellungen im Norden der Iberischen Halbinsel zusammen, die merkwürdigerweise nie von den Quellen widergespiegelt werden. ${ }^{7}$

5. Die Literatur zu Priscillian ist sehr umfangreich, siehe R. M. SAnz SERrano: «Aproximación al estudio de los ejércitos privados en Hispania durante la Antigüedad Tardía», Gerión, 4 (1986), 225-264 und Dies.: Paganos, adivinos y magos, 139ff. Sulpicius Severus (II, 1ff) wurde als Häretiker betrachtet, mit dem Gnostizismus und Manichäismus zusammenhängend (Hieronymus, epist. 45,3-4 und epist. 120, 2, 10). Ebenso in Priscillians Tract., I, 15-20. In Wahrheit handelte es sich jedoch um einen Konflikt zwischen den spanischen Aristokratien. Siehe E. Сн. Babut: Priscillien et le priscillianisme, Paris, 1909; H. CHADwiCK: Prisciliano de Ávila, Madrid, 1978 und V. E. PAÑo: Iglesia y estado en el certamen priscilianista. Causa ecclesiae y iudicum publicum, Zaragoza, 1988.

6. Sulpicius Severus II, 46, 6: [...] familia nobilis, praedives opibus, acer, inquies, facundus, multa lectione eruditus, disserendi ac disputandi promptissimus, felix profecto, si non pravo studio corrupisset optimum ingenium; prorsus multa in eo animi et corporis bona cerneres. Vigilare multum, famem ac situm ferre poterat, habendi minime cupidus, utendi parcissimus. Sed idem vanissimus et pluro iusto inflatior profanarum rerum scientia; quin et magicas artes ab adolescentia eum exercuisse creditu es; Hieronymus, epist. 133, 3 (ad Ctesiphontem): In Hispania Agape Elpidium, mulier virum, caecum caeca duxit in foveam, successoremque sui Priscilianum habuit, Zoroastris magi studiosissimum, et ex mago episcopum.

7. Priscillian wurde wegen Hexerei verurteilt, vgl. Sulpicius Severus II, 50, 1-8: Is Priscilianus gemino iudicio auditum, convictumque maleficii nec diffitentem obscoenis se studuisse doctrinis, nocturnos etiam turpium foeminarum egisse conventus, nudumque orare solitum, nocentem pronuntiavit redegitque in custodiam, donec ad Principem referret. Priscillian und seine adeligen Anhänger wurden enthauptet. Pacatus Paneg. Lat., XII, 25 bestätigt, dass sie von Dieben verurteilt wurden: Fuit enim, fuit et hoc delatorum genus qui nominibus antistites, se vera autem satellites atque adeo carnifices, non contenti miseros evoluisse patrimoniis calumniabantur in sanguinem et vitas premebant reorum iam pauperum [...]. Aber sie wurden immer als Häretiker betrachtet (Orosius, Comm., 2; Augustinus, Contra Mendacio, III, 5 und epist. 137; Hydatius, Chron., 16, 102 und 135 und C. Th., XVI, 5, 40-65). Siehe C. Cardelle De Hartmann: «El priscilianismo tras Prisciliano ¿un movimiento galaico?», Habis, 29 (1998), 269-290. 
Es ist überraschend, dass in den ersten Jahren des 5. Jahrhunderts und trotz des Hiatus, den die Einwanderung germanischer Gruppen in diese Gebiete verursachte, ausschließlich von einer Hispania die Rede ist, die zum Heidentum zurückkehrte, aber nicht von einer heidnisch gebliebenen Hispania, und zwar sowohl in den Hauptwerken von Hydatius und Orosius, als auch in den nachrangigen Berichten von Pacanianus von Barcino oder Licinianus Carthaginensis. Selbst in seiner Erzählung über die Bekehrung der Einwohner Mahons bezieht sich Bischof Severus nur auf die jüdische Gemeinschaft der Insel. Nachdem Teodoro, der wichtigste Händler der Insel und patronus der Gemeinde, konvertierte, brachte er plebem suam mit sich. ${ }^{8}$

Das Wiedererstarken des Paganismus im 6. Jahrhundert erscheint paradox, weil es mit der Festigung der suebischen und westgotischen Monarchien zusammenfällt. Hinsichtlich der Sueben bezeugen dies der Mönch Martin von Braga und die Konzilien von Braga aus den Jahren 561 und 572, hinsichtlich der Westgoten das dritte Konzil von Toledo, welches im Jahr 589 von Rekkared einberufen wurde. In beiden Fällen findet man das Heidentum tief verwurzelt, nicht nur im fernen Norden, welcher von keiner dieser Monarchien beherrscht wird, sondern überall in ganz Hispania, wie das toledanische Konzil urteilt: quoniam pene per omnem Spaniam idolatriae sacrilegium inolevit (can. 16). Die Unterdrückung des Paganismus wird in den Konzilien und Gesetzen den Herren der fundi und den Bischöfen und Richtern in den Städten aufgetragen, indem man die Vernichtung (exterminata truncentur) der Irrtümer des Pöbels (errores plebium) fordert und die Scharfrichter als exquisitores, cultores und extirpadores benennt. ${ }^{9}$ Als Gründe für die Existenz des Paganismus werden im tomus des XI. Konzils von Toledo (can. 12) aus dem Jahr 675 die allgemeine Gleichgültigkeit, die Unwissenheit und die Sünde angegeben sowie, letzten Endes das Fehlen von konkreten Aktionen dagegen per omnem Spaniam sive Galliam, was selbst auf dem von Egica einberufenen XVI. Konzil von Toledo aus dem Jahr 693, dem letzten vor der Ankunft der Mauren, noch so gesehen wird. Das steht im Gegensatz zur Patristik, die alles, was mit dem Heidentum zu tun

8. Severus, ep. 5,1. Über das patrocinium: III. Konzil von Toledo, can. 14; IX. Konzil von Toledo, cans. 5 und 6; XVI. Konzil von Toledo, can. 5; IV. Konzil von Toledo, cans. 49, 53, 60, 61, 69; Konzil von Sevilla, cans. 1, 2, 10 und 11, in: R. M. SAnz Serrano: «Las relaciones de dependencia como factor de cristianización en la Península Ibérica: grupos de edad y cristianización», in: M. M. Myro, J. Alvar D. Plácido (Hg.): Las edades de la dependencia durante la Antigüedad, Madrid, 2000, 395-424 und R. GonzÁLEz SALINERO: «Relaciones sociales y dependencia religiosa en la comunidad judía de Mahón (Menorca) a principios del siglo v d. C.», Arys, 3 (2000), 267-277.

9. Cans. 16 des III. Konzils von Toledo, 11 des XII., 2 des XVI. und 14 des Konzils von Narbona. R. M. Sanz Serrano: «La excomunión como sanción política en el reino visigodo de Toledo», Antigüedad y Cristianismo, III (1986), 275-288 und Dies.: «Paganos, adivinos y magos», 97 f. Außerdem J. MartíNEZ Díez, F. Rodríguez: La colección canónica hispana, Madrid, 1984; P. D. KIng: Derecho y sociedad en el reino visigodo, Madrid, 1981 und R. W. CARLYLE: A history of Mediaeval Political Theory in the West, Edinburg, 1950. Über das Problem in Baetica: F. SAlvador Ventura: «La iglesia bética a finales del s. VI y comienzos del vir. Cuestiones políticas y socioeconómicas», in: J. Alvar (Hg.): Homenaje a J. M. Blázquez, 1994,, 229-240 und P. U. RabAneda: La iglesia en la Hispania del siglo V, Granada, 2004. 
hat, ignoriert oder, wie Isidor, als ein Relikt der Vergangenheit darstellt. Die Vita Sancti Fructuosi Bracarensis episcopi von Valerius und die Vita Sancti Aemiliani aus dem 7. Jahrhundert bringen die Heiden mit den nördlichen Gebieten in Verbindung, die überwiegend nicht von der gotischen Monarchie kontrolliert werden. ${ }^{10}$ Die Quellen verweisen dort bis zum 5. Jahrhundert auf hispanische bagauda und später (Biclarensis) auf heidnische und barbarische vascones und rucones, die christliche Dörfer und Städte in der Nachbarschaft angriffen, und zwar im Kampf gegen den Staat. Leute unterschiedlichster Herkunft waren in bacaudam organisiert und in ländlichen Grenz- und Randgebieten konzentriert und wollten nicht weiter römisch sein, worüber Salvian von Marseille in seinem De gubernatione Dei (V, 21-26) berichtet. Hier trafen sich all diejenigen, die nicht nur politisch, sondern auch religiös verfolgt wurden und welche man im Jahr 632 - laut der Vita Amandi des Baudemundus - immer noch erfolglos zu christianisieren versuchte. ${ }^{11}$

Die schriftlichen Quellen scheinen weit von diesen Ereignissen entfernt und verstümmeln die Informationen, reißen sie auseinander und verdrehen sie. Jeder Versuch, die heidnischen Glaubensrichtungen zu klassifizieren, ist zum Scheitern verurteilt. Am stärksten waren die Religionen griechischen und orientalischen Ursprungs betroffen, die in römischer Zeit einen Synkretismus erlebten, wie zum Beispiel der Herkules-, Melkart- oder Asklepios-Kult. Auch die orientalischen Mysterienkulte werden nicht erwähnt, obwohl ihnen nach Aussage der epigraphischen Angaben in denjenigen römischen Städten eine gewisse Bedeutung zukam, deren Bevölkerung aus Militärund Geschäftsleuten bestand. Diese verfügten sogar über Tempel zu Ehren der Isis in Baelo und Italica, zu Ehren von Mithras in Emerita, Italica und Troia sowie über das taurobolium der Kybele bei Monte Cildá in Palencia. Außerdem kamen sie dazu, sich mit einheimischen Göttern in Panoias zu vereinigen. Man nahm an, dass die Lehre und Botschaft von der Universalität dieser Religionen, die eng mit der Staatsideologie verbunden sind, mit der Christianisierung derselben hätten verschwinden können. ${ }^{12}$ Abgesehen von den Verfolgungen ist in diesem Zusammenhang wichtig, dass in Carmona bei Sevilla bei der Tumba del Elefante ein mutmaßliches Kybele-Heiligtum aus

10. S. Castellanos: Historiografía y sociedad en la Hispania visigoda. La vita Aemiliani y el actual territorio riojano (siglo VI), Logroño, 1999; DERS.: La hagiografía visigoda. Dominio social y proyección cultural, Logroño, 2004.

11. Über die bagauda R. M. Sanz Serrano: Historia de los Godos. Una epopeya histórica de Escandinavia a Toledo, Madrid, 2009; A. BArbero, M. Vigil: Sobre los orígenes sociales de la Reconquista, Barcelona, 1974; C. SÁnchez Albornoz: Vascos y navarros en su primera historia, Madrid, 1976 und J. C. SÁNCHEz LEón: Los bagaudas: rebeldes, demonios, mártires. Revueltas campesinas en Galia e Hispania durante el Bajo Imperio, Jaén, 1996 und R. Collins: Los vascos, Madrid, 1989.

12. Zu Panoias: G. AlfÖLDY: «Inscripciones, sacrificios y misterios: El santuario rupestre de Panoias, Portugal», MM, 36 (1995), 252-258. Zu den Mysterien in Hispania: J. Alvar, C. Blánquez, C. G. Wagner (Hg.): Sexo, muerte y religión en el mundo clásico, Madrid, 1994 und R. RuBio (Hg.): Isis. Nuevas perspectivas, Madrid, 1981. Allgemeiner: J. Alvar: Los misterios. Religiones „orientales“ en el Imperio romano, Barcelona, 1992; W. Burkert: Ancient Mistery Cults, Massachussets, London, 1987 und R. Turcan: Les cultes orientaux dans le monde romain, Paris, 1989. 
dem 4. Jahrhundert absichtlich verstümmelt wurde, welches mit Statuen von Atis, einem Elefant und einem betilus ausgestattet gewesen war. ${ }^{13}$

Im Gegensatz dazu verfügen wir über Schriftzeugnisse zu römischen Kulten, die bis in das 6. Jahrhundert reichen, wobei es vielfach zu einer Vereinigung mit einheimischen Göttern gekommen ist. Doch die Anspielungen auf den Kapitols- oder Kaiserkult hören mit dem frühen Konzil von Elvira im 4. Jahrhundert auf (cs. 2, 3, 56 und 59), was angesichts des Wechsels der Staatsideologie selbstverständlich scheint. Stattdessen überleben eher private Kulte, vor allem für die kleinen Götter wie die Nymphen, Lamien und Laren, die auch einheimische Wurzeln haben. Im 4. Jahrhundert merkt Priscilian in dieser Hinsicht an, dass er und seine Anhänger beschuldigt würden, an Jupiter, Mars, Mercur, Venus und Saturn zu glauben, sowie Tiere zu verehren (Tract., I, 15). Mehr als ein Jahrhundert später berichtet Martin von Braga für dieselben nördlichen Länder unter suebischer Herrschaft wieder von der Verehrung von Neptun, der Lamia, von Nymphen und der Diana, von den vertriebenen Dämonen des Himmels, welche die Seen, Quellen, Ströme und Wälder bewohnen. ${ }^{14}$ Ein Jahrhundert danach erscheinen diese Götternamen im enzyklopädischen Werk des Isidor von Sevilla. Dieser richtete sich an den christlichen Adel, um ihn zu warnen und den Ursprung dieser Kulte zu erläutern, die in den Gesetzen und Konzilien zusammen mit Resten einheimischer Götter zu Götzenverehrung herabgestuft wurden. Die Mosaiken einiger Villen des 5. bis 6. Jahrhunderts bestätigen trotz der Christianisierung der Kunst ein gewisses Fortleben mythologischer Themen und Stereotype, hauptsächlich dionysischer Motive. ${ }^{15}$

Anders verhält es sich mit den einheimischen Göttern, für die literarische und mythologische Dokumente fehlen, die bereits im römischen Zeitalter rar sind. Die Götternamen in epigraphischen Quellen beziehen sich zum größten Teil auf heidnische Gottheiten in den nördlichen und westlichen Gebieten, von denen einige mit römischen Göttern vereinigt sind, was es erlaubt, Überlegungen zu ihrer Bedeutung anzustellen (Dulovius von Caceres, Bormaricus von Caldas de Vizella, Vurovius von La Bureba, Mars Tilenus von Quintana del Marco, Iupiter Candamius von Candanedo in Leon, genius Laquiniensis von S. Miguel de Vizella oder Ataecina/Proserpina von

13. M. Bendala Galán: La necrópolis romana de Carmona (Sevilla) 1976 und Ders: «Las religiones mistéricas en la España romana», Symp. Religiones romanas en Hispania, Madrid, 1981, 285-297.

14. De correct. 6: Praeter haec autem multi daemones ex illis qui de caelo expulsi sunt, aut in mari, aut in fluminibus, aut in fontibus, aut in sylvis praesident, quod similiter homines ignorantes Dominum, quasi Deos colunt, et sacrificant illis, et in mari quidem Neptunum appellant, in fluminibus Laminas, in fontibus Ninfas, in silvis Dianas, quae Omnia maligni daemones et spiritus nequam [...].

15. Wie die von den Marroquíes Altos (Jaén), Mérida, Arróniz und Liédana (Navarra), Alcalá de Henares, Albadalejo (Ciudad Real), Baños de Valdearados (Burgos), Torre de Palma (Monforte, Portugal) u.v.a. In J. M. Blázquez Martínez: Romanos de España, Barcelona, 1993; M. Guardia Pons: Los mosaicos de la Antigüedad Tardia en Hispania. Estudios de iconografía, Barcelona, 1992. Über den Adel in Spanien, R. M. Sanz Serrano: «Aristocracias paganas en Hispania Tardía (s. V-VII)», in: D. Plácido Suárez, F. J. Moreno Arrastio, L. A. Ruíz Cabrero (Hg.): «Necedad, sabiduría y verdad: el legado de Juan Cascajero», Gerión, 25, 1, 2007, 443-480. 
Extremadura). Trotzdem gehören diese Inschriften spätestens an den Anfang des 4. Jahrhunderts, und selbst das jüngste Exemplar, der Altar für Erudinus auf dem Gipfel von Dobra in Santander aus dem Jahre 399, wird heutzutage für älter gehalten. ${ }^{16}$ Zwischen den jüngsten epigraphischen Zeugnissen vor Einführung des Christentums und dem Höhepunkt der späteren christlichen Epigraphik besteht ein beträchtlicher Hiatus. Ihr Verschwinden wird kaum durch christliche Weihungen ausgeglichen, die erst dann häufiger erscheinen, als Inschriften $\mathrm{zu}$ einem bedeutenden Mittel politischer Propaganda des westgotischen Staates werden. Doch einheimische Götter tauchen auch in der Patristik nicht auf, ausgenommen bei Priscilian in den Anspielungen an Sonne und Mond sowie an Gottheiten, die Donner, Gewitter und Dürren hervorbringen, wie sie auf den concilia bracarensis im 6. Jahrhundert erwähnt werden. ${ }^{17}$ Sie kommen auch nicht in den Anschuldigungen des Braulius und Valerius im Norden der Halbinsel vor. Sie erscheinen ferner überhaupt nicht im Werk des Isidor, so als ob die Götter der gentilitates einer anderen, von der klassischen Kultur fremden Vergangenheit angehörten. Es sind die Konzilien, die uns vermuten lassen, dass sie sich in den ursprünglichen Formen der Verehrung von Naturmächten, im Kult von Götzen, Steinen, Bäumen, Quellen oder im Feuer der Fackeln (cultores idolorum, venatores lapidum, accensores facularum et excolentes fontium vel arborum ${ }^{18}$ ) verbergen. Dieses Vorgehen finden wir schon bei Martin von Braga. Es zielt darauf ab, die hohe Anzahl von Göttern der hispanischen Bevölkerung nicht nennen zu müssen. ${ }^{19}$

Das Fehlen konkreter Zeugnisse für alle Arten von Gottheiten und ihre Begrenzung auf natürliche Orte führen zum Verschwinden der Altäre und Tempel. In der Tat enthält der Codex Theodosianus (XVI, 10, 15) ein spezifisches Gesetz für Hispanien aus dem Jahre 399, in dem die Schließung der Tempel und die Verwahrung ihrer Ausstattung angeordnet wird: publicorum operum ornamenta servari. ${ }^{20}$ Eine Zerstörung könnte schon etwas früher nach dem Konzil von Elvira begonnen haben. Dieses Phänomen war weit verbreitet, wie die Taten von Martin von Braga und Valerius aus dem 6. bis 7. Jahrhundert oder die westgotischen Gesetze zur Wiederbenutzung von

16. Gewidmet von Cornelius vicanus Aunigainum Cesti Filius im Konsulat von Manlius und Eutropius. A. García y Bellido: „Tres piezas del Museo Arqueológico Provincial de Santander“, AEspA, 22, 1949, 241. Neulich, L. J. MacLennan: «Nota sobre la inscripción latina del ara votiva a „Erudino“ de la Cantabria romana», AespA, 69, 1996, 311-14. Für die kürzliche Spätdatierung vgl. A. ST YLOw, CIL II 5809.

17. Priscilian Tract., I, 4. Außerdem I. Konzil von Bracara, can. 9 und II. Konzil von Bracara, can. 72.

18. Can. 11 vom XII. Konzil von Toledo

19. De correct.,7: Nam ad petras et ad arbores et ad fontes et per trivia cereolos incendere, quid est aliud nisi cultura diaboli. Can. 11, XII. Konzil von Toledo: Sed cultores idolorum, venatores lapidum, accesores facularum et excolentes fontium vel arborum admonemus, ut agnoscant quod ipsi se spontanae morti subiciunt qui diabolo sacrificari videntur [...]. Can. 2, XVI. Konzil von Toledo: Illi diversis suadellis decepti cultores idolorum efficiuntur, veneratores lapidum, accensores facularum, excolentes sacra fontium vel arborum, auguratores quoque seu praecantatores quoque seu praecantatores, multaque alia quae longum est enarrare.

20. Honorius et Arcadius für Macrobius vicario Hispaniarum et Procliano vicario quinque provinciarum: Sicut sacrificia prohibemus, ita volumus publicorum operum ornamenta servari. Ac ne sibi aliqua auctoritate blandiantur, qui ea conantur evertere, si quod rescriptum, si qua lex forte praetenditur. 
Spolien aus Gräbern und Mausoleen bestätigen (Cod. Theod. IX, 7, 1-7; Leges Visigothorum, XI, 2,1-2). ${ }^{21}$ Direkte Belege sind ferner die Zerstörung der schon erwähnten Tumba del Elefante und von Kirchen wie Fructuoso de Montelios in Braga oder des martyrium von Marialba. Dagegen ist es im Allgemeinen schwer, diese Zerstörungen heute noch in den Städten archäologisch ausfindig zu machen, da sie mehrheitlich weiterbewohnt wurden. Überdies wissen wir aus Fällen wie Corduba oder Emerita, dass dort die ersten christlichen Gebäude bis in sehr späte Zeit eher Randgebiete einnahmen und gleichzeitig neben den alten öffentlichen und religiösen Plätzen bestanden. Selbst in Toletum hat man noch keine Spuren der Christianisierung entdeckt. Daneben werden neuerdings vermehrt alte Datierungen in Frage gestellt, die mehr auf literarischen Texten als auf archäologischen Befunden beruhen, wie etwa im Fall des Baptisteriums von Barcelona oder der Kirche im Amphitheater von Tarragona, die beide auch jünger sein könnten. ${ }^{22}$

Genau so vielschichtig und komplex ist die Christianisierung des ländlichen Raumes, wo die Forschung dazu neigte, das Christentum mit dem Auftreten von Basilika-artigen Grundrissen in Villen zu verknüpfen. Das Ende dieser Villen datierte man wiederum mit dem ersten Auftreten germanischer Völkerschaften im 5. Jahrhundert. Diese Sichtweise beruhte auf alten Ausgrabungen und sehr fraglichen typologischen Analysen. Deshalb bemühen sich neuerdings Wissenschaftler wie Caballero Zoreda, ${ }^{23}$ eine Überprüfung dieser Datierungen durchzuführen. Als Belege für eine Christianisierung fordern sie andere Beispiele, nicht nur Basilika-artige Grundrisse. Man hält es sogar für möglich, dass die späteren Kirchenbauten im Norden der Halbinsel gar nicht westgotisch, sondern vielleicht sogar aus der Umayyadenzeit sein könnten. Demselben Problem begegnen wir bei mittelalterlichen Kirchen, die an Plätzen errichtet wurden, von denen in großer Zahl Inschriften mit Widmungen an heidnische Gottheiten bekannt sind. Da dort Ausgrabungen fehlen, lassen sich weder Kontext noch Chronologie näher beurteilen.

21. Außerdem can. 46 des IV. Konzil von Toledo des 633: Si quis clericus in demoliendis sepulchris fuerit deprehensus, quia facinus hoc pro sacrilegio legibus publicis sanguine vindicatur, oportet canonibus in tali acelere proditum a clericatos ordine submoveri et poenitentiae trienium deputari.

22. J. M. Gurt y Esparraguera, G. Ripoll y C. Godoy: «Topografía de la Antigüedad Tardía hispánica. Reflexiones para una propuesta de trabajo», Ant. Tard., 2, 1994, 161-180; P. Mateos Cruz: «Arqueología de la tardoantigüedad en Mérida: estado de la cuestión», in: I. VelázQuez, A. Cerrillo, P. Mateos: «Los últimos romanos en Lusitania, Cuadernos Emeritenses», 1995,126-149; J. ArCE: «Mérida Tardorromana (300-580 d.C)», Cuadernos Emeritenses, 22, 2002; A. MARCONE: «La cristinización de la civitas en Occidente», in: J. SAntos, R. Teja (Hg.): «El cristianismo. Aspectos históricos de su origen y difusión en Hispania», Revisiones de Historia Antigua, III, 2000, 37-52; R. SAnz Serrano, Historia de los Godos, 377

23. Zum Beispiel El Trampal (Cáceres), S. Pedro de la Mata (Toledo), S. Pedro de la Nave (Zamora), S. Juan de Baños (Palencia), Quintanilla de las Viñas (Burgos), L. CABAllero Zoreda: «La arquitectura denominada de época visigoda ¿es realmente tardorromana o prerrománica?», in: L. CABALLERO, P. Mateos (eds.): Visigodos y Omeyas. Un debate entre la Antigüedad Tardía y la Alta Edad Media, Madrid, 2000, 207-247. Dagegen in derselben Publikation A. Arbeiter: «Alegato por la riqueza del inventario monumental hispano-visigodo», 249-263. Das Problem sind die fehlenden archäologischen Ausgrabungen in den Kirchen. 
Da aber den Göttern ihre Namen, ihr Eigentum und ihr Priestertum geraubt wurden, bleiben uns nur die Hinweise auf anonyme Götterkulte. Gebete, Libationen, Opfer und Gaben ohne einen bestimmten Adressaten und vor allem Festtage wie Kalendas, Vulcanalia und Paganalia sind auf die eine oder andere Art in den Quellen des 4. bis 7. Jahrhunderts gegenwärtig. ${ }^{24}$ Hinzu treten christliche Feiertage, die ältere Feiertage heidnischen Ursprungs ersetzten, wie zum Beispiel Ostern, und die im can. 73 von Braga noch als observatio paganismi bezeichnet werden. Außerdem behauptet Salvian von Marseille (De gub. Dei, VI, 12, 69), die Kirchen würden in jener Zeit immer leerer und der Zirkus immer voller (ecclesia vacuatur, circus impletur).

Zuletzt sind noch zahlreiche Anklagen gegen Wahrsagerei und Zauberei zu erwähnen, verbunden mit unterschiedlichen religiösen Handlungen, vor allem kombiniert mit Opfern an die Götter, und zwar wieder im Gegensatz zwischen dem Wahren und dem Falschen in den Religionen, wobei das Heidentum wieder sehr abwertend dargestellt wird. Dies begegnet uns in der römischen wie in der westgotischen Gesetzgebung und von den Kanones des Konzils von Elvira bis hin zu den Schriften Isidors. Die westgotischen Konzilien sind voller Anklagen gegen verschiedene Magien und Verehrungsformen, augures, sortilegi, divini, mathematici, aruspices, astrologi und vaticinatores, die im Untergrund arbeiten. Sie stimmen mit Bezeichnungen in den Etymologiae des Isidor überein, die von einem klassischen Modell der verschiedenen disciplinae der divinatio ausgehen. ${ }^{25}$ Auf diese Weise wiederholen sich bei Isidor (Etym. VIII, 9, 9ff) die necromantii, hydromantii, geomantii, aeromantii, pyromantii, arioli, divini, incantatores, aruspices, augures, pythonissae, astrologi, sortilegi und salisatores, ohne dass wir diese Einteilung in der Praxis wiederfinden. Sie gelten nicht als einfache Anmerkungen eines Gelehrten, sondern als real auftretende Praktiken, die auf den von den Monarchen geleiteten Versammlungen der Konzilien debattiert und verfolgt wurden (can. 2 des XVI. Konzils von Toledo). Einige dieser Glaubensrichtungen sind bei Christen ausführlich bezeugt, wie etwa bei den Chronisten Hydatius und Orosius, wenn sie über außergewöhnliche Phänomene berichten. Daneben

24. Cans. 2, 3, 40, 41 des Konzils von Elvira. Licinianus von Carthagena, ep. Ad Vicentius, III, 2: Melius erat viro hortum facere, iter agüere, mulieri colum tenere, et non ud dicitur, ballare, saltare, et membra a deo bene condita saltando male torquere, et ad excitandam libidinem nugatoribus cancionibus proclamare. Pacianus von Barcino, Paraenesis, II; Martin von Braga, De correct. 7: Vulcanalia et Kalendas observare, mensas ornare, et lauros ponere, et pedem observare, et fundere in foco super truncum frugem et vinum, et panem in fontem mittere, quid est aliud nisi cultura diaboli?. Außerdem in can. 23, III. Konzil von Toledo: ut populi qui debent officia divina adtendere saltationibus et turpibus invigilent canticis, non solum sibi nocentes sed et religiosorum officiis prestrepentes [...].

25. Cans. 6, 14, 15 Narbona, 29 vom IV., 4 vom V., 11vom XII., 2 und 8 vom XVI., 7 vom XVII. Konzil von Toledo. Isidorus, Etym., VIII, 9, 1: Magi sunt, qui vulgo maleficio ob facinorum magnitudinem nuncupatur. Hi et elementa concutiunt, turbant mentes hominum, ac sine ullo veneni haustu violentia tantum carmines interimunt. Gleichfalls Lex Visigothorum, VI, 2, 1. 
benutzen sie fulgura oder exta, um historische Ereignisse als Werke der Götter zu erklären. ${ }^{26}$

Allgemein gesprochen gibt es keine spezifischen Merkmale, die bei der Untersuchung verschiedener religiöser Verehrungsformen in der hispanischen Spätantike exklusiv auftreten. Dies gilt sowohl für die Zeugen von Wundern als auch für die incantatores, die das Wort als Mittel benutzten, außer bei den vana carmina, die von den Konzilien verfolgt wurden (I. Konzil von Bracara, can. 12; II. Konzil von Bracara, can. 59, IV. Konzil von Toledo; can. 11; XVI. Konzil von Toledo, can. 2). So können wir die tabulae defixiones als magische Rituale betrachten, die über die ganze Halbinsel verbreitet sind, bis ins Mittelalter reichen und zur heidnischen wie christlichen Religionsausübung gehören. Ähnliche Zauberformeln sind der Göttin Ataecina sowie christlichen Heiligen gewidmet. ${ }^{27}$ Wir wissen auch wenig Konkretes über die necromantii, die die Toten durch komplizierte Zeremonien wieder auferstehen ließen. Um sie zum Sprechen $\mathrm{zu}$ bringen, injizierten sie ihnen eine Mischung aus Blut und Wasser. ${ }^{28}$ Möglicherweise verweist can. 35 von Elvira auf diese Praxis, denn dort ist davon die Rede, dass den Frauen das Übernachten auf Friedhöfen verboten wird. Ebenso haben wir keine konkreten Angaben zu den salisatores ex membrorum quaecumque partes salierin, die mit Teilen des menschlichen Körpers wahrsagen. Entsprechendes gilt für die sortilegi, die Isidor mit Schriftbüchern, mit der Kabbala und mit den sanctorum sortes verbindet. ${ }^{29}$ Ferner fehlen uns konkrete Fälle, die von astrologi und mathemateci berichten, deren Aufgabe es war, Horoskope anzufertigen und die Zukunft aus den Sternen vorauszusagen. Bis zum 6. Jahrhundert bilden die Beschuldigungen gegen die Priscilianer unsere einzige Quelle. Angeblich setzten sie heidnische Traditionen fort, die auf himmlischen Wesen und auf der Beobachtung von Mond und Sternen beruhten. Diese Rituale kamen zur Anwendung, wenn ein Haus gebaut, wenn gesät oder wenn eine Ehe geschlossen wurde..$^{30}$ Nicht viel mehr wissen wir über die schon erwähnten haruspices, auguratores und augures, die sich bei Isidor und auf

26. fulgura oder exta benutzt für die Rechtfertigung des Werk Gottes von Orosius, Hist. V,18,1; VII,15,12 und Hydatius, Chron.,34, 48, 64, 136, 139 Erdbeben, Finsternissen und Kometen. S. MonTERO: Trajano y la adivinación. Prodigios, oráculos y apocalíptica en el Imperio romano, Anejos Gerión, IV, 2000 und R. Bloch: Prodigi e divinazione nel mondo antico, Roma, 1981.

27. I. VelázQuez: Las pizarras visigodas. Entre el latín y su disgregación. La lengua hablada en Hispania, siglos VI-VIII, Burgos, 2004.

28. D. Pérez Fernández, M. Seguido: «Suscitación de cadáveres con fines adivinatorios en el mundo romano», in: J. Alvar, C. Blánquez, C. G. WAgner (Hg.): Sexo, muerte y religión en el mundo clásico, 1994, 171-192.

29. Es gibt einen Hinweis im II. Konzil von Bracara, can. 71: Si quos paganorum consuetudinem sequens divinos et sortilegos in domo sua introduxerit, quasi ut malum foras mittant aut maleficia invaniant vel lustraciones paganorum faciant, quinque annis poenitentiam agant.

30. Can. 72, II. Konzil von Bracara: Non liceat christianis tenere traditiones gentilium et observare et colere elementa aut lunam aut stellarum cursum aut inanem signorum fallaciam pro domo facienda vel segetes vel arbores plantandas vel coniuge socianda. 
den Konzilien kontinuierlich wiederholen. ${ }^{31}$ Sie waren damit beschäftigt, die exta oder die Eingeweide der Opfer zu untersuchen, oder, laut Isidor, die Flüge und Stimmen der Zugvögel (auguria qui volatus avium et voces intendum) auszulegen: Die iniquas observationes der Heiden des can. 73 des II. Konzils von Braga. Sie kommen ferner in den Abhandlungen der Priscilianer und bei Martin von Braga vor, der sich über diese Praktiken bei den Feierlichkeiten beklagt. Wenig lässt sich über die arioli sagen, die mit Statuen prophezeiten; sie werden nur in den Bemerkungen der Konzilien zum Götzendienst erwähnt (cans. 11 und 59 von Braga I; III. Konzil von Toledo, can. 16 und XVI. Konzil von Toledo, can. 2).

Ein Beispiel für das Weiterleben eines solchen Rituals bietet die incubatio, also die Heilung durch die onéroi, worüber in der Antike die Abhandlungen des Lydiers Artemidorus und des Christen Synesius von Kyrene berichten. Dieses Ritual praktizierte man in Tempeln, wo es Fachmänner gab, deren Aufgabe es war, Kranke zu heilen, und zwar durch die Botschaften, die die Götter durch die Träume sandten. Diese Tempel konnten auch den Orakeln gewidmet sein, wie das zum Beispiel in den Heiligtümern für Melkart in Gades, Äskulap in Ampuries, Jupiter in Clunia und Isis oder Serapis vorkam. ${ }^{32}$ In Hispanien kann man den Wandlungprozess an zwei einheimischen Sanktuarien verfolgen, die die gesamte römische Zeit über in Betrieb waren, beide in Lusitanien. Das erste ist der Göttin Turibringesis Ataecina gewidmet, die mit der Proserpina in der heutigen Santa Lucía del Trampal nahe Emerita Augusta in Verbindung stand. Das Heiligtum befindet sich in einer bewaldeten Ebene mit heiligen Quellen sowie an einem privilegierten Ort an der Via de la Plata gelegen. Von dort kommen viele Weiheinschriften mit den Formeln ex voto, votum solvit und ex visu. Aber der Synkretismus wird durch die Verbindung mit der heiligen Lucia sichtbar. Ihr werden Heilkräfte zugeschrieben. Die Datierung steht allerdings aufgrund der Ergebnisse, die der Ausgräber Caballero aufgeworfen hat, in Zweifel. Er hat die Ermita Santa Lucía del Trampal, die mit den Spolien des ehemaligen heidnischen Tempels erbaut wurde, in die Umayyadenzeit datiert. ${ }^{33}$

Ein ähnlicher Fall liegt mit einem Heiligtum des Endovelicus in Portugal vor, in San Miguel de la Mota in Alandroal. Endovelicus war in der römischen Zeit einer der wichtigsten eingeborenen Götter der Halbinsel. Die mittelalterliche Ermita San Miguel, ein anderer Heiliger mit Heilkräften, brachte bei

31. Can.71 des II. Konzil von Bracara: Si quos paganorum consuetudinem sequens divinos et sortilegos in domo sua introduxerit". Martin von Braga, De correct.,7"Divinationes et auguria et dies idolorum observare und 12 Non iussit Deus hominem futura cognoscere, sed ut semper in timore illius viveret;IV. Konzil von Toledo, can. 29 Si episcopus quis aut prsbyter sive diaconus vel quilibet ex ordine clericorum magos aut aruspices aut ariolols aut certe augures vel sortilegos vel eos, qui profitentur artem aliquam, aut aliquos eorum similia exercentes, consulere fuerit deprehensus, ab honore dignitatis suae depositus monasterii und XII. Konzil von Toledo, can. 11.

32. Über den incubatio, P. Cox Miller: Dreams in Late Antiquity. Studies in Imagination of a Culture, Princeton, 1994; N. Fernández Marcos: Los Thaumata de Sofronio. Contribución al estudio de la incubatio cristiana, Madrid, 1975; M. A.VInAgRe Lobo: Los intérpretes de sueños en los templos de Serapis, Arys, 3, 2000, 125-144.

33. L. Caballero Zoreda: La arquitectura denominada (wie Anm. 23). 
Ausgrabungen Statuen römischer Zeit ans Licht, die offenbar absichtlich schwer beschädigt wurden. Es gibt zahlreiche weitere unausgegrabene Heiligtümer mit exvotos oder mit Inschriften pro salute und ex visu zum Dank für Heilungen. Ihre Namen wurden später christianisiert, und an ihren Orten errichtete man danach christliche Kirchen wie in Santa Eulalia de Bóveda bei Lugo oder Santa Lucía del Trampal in Cáceres. ${ }^{34}$ Daneben kennen wir auch christliche Heiligtümer, die auf Heilungen spezialisiert waren, wie zum Beispiel das Heiligtum der Eulalia in Emerita Augusta mit einem Altar mit drei Bäumen, deren Blüten heilten, dazu mit einem Priesterstand, der damit beauftragt war, die Blumen im xenodochium des Klosters zu verteilen (Gregor von Tours, L. M. I, 91; Vitas Patres Emeritensium 5, 3, 8-15).

Abschließend sei festgehalten, dass nicht die Patristik, sondern die Konzilien und späteren Gesetze innerhalb der verschiedenen heidnischen Aktivitäten von Männern und Frauen als vir ac mulieres divinatores (Konzil von Narbonensis, can. 14), sowohl von ingenui als auch von servi (V. Konzil von Toledo, can. 4), cuiuscumque sint generis aut conditiones, das heißt, an jedem Ort, von jedem Geschlecht und jeder Wesensart, und in ganz Hispanien und Gallien sprechen. Im Konzil von Narbonensis aus dem Jahr 589 (can. 14) heißt es, dass der Paganismus in Hispania und Gallia von gothi, romani, siry, graeci vel iudaei ausgeübt wird. ${ }^{35}$ Die Leges Visigothorum (VI, 2,1-5) nennen die Heiden erroris spiritu pleni, und zwar Freie wie Sklaven (ingenui, hominis, servi) beiderlei Geschlechts und mit verschiedenem Rechtsstatus (sue ingenuus sit, sive servus utriusque sexus) sowie jedes Standes (cuiuslibet sit ordinis vel persone). Es handelt sich um unterschiedlichste Menschen mit einem noch sehr lebendigen heidnischen Brauchtum, die die Patristik als Christen betrachtete. Als ihre Heiligtümer verschwunden waren, wirkten sie an jenen Orten weiter, von denen - nach Martin von Braga - die Götter vertrieben wurden. Aber sie praktizierten ihre Rituale auch zum Schutz der Häuser, der Natur und der Kreuzungen. Manche dieser Orte waren schon von einer christlichen Kirche besetzt. Aus diesem Grund sprechen Isidor, die Konzilien und Gesetze nicht von Heidentum, sondern von Götzenverehrung, Zauberei und Wahrsagerei, die auf diese Weise nur mehr als letzte Äußerungen eines heidnischen Priesterstandes zu gelten hatten, dessen Mitglieder später mit Schwätzern und Hexenmeistern gleichgesetzt wurden. ${ }^{36}$

34. R. Sanz Serrano: «La destrucción de centros de culto paganos como forma de persecución religiosa en la Península Ibérica», in: J. Alvar (ed.): Homenaje a J. M. Blázquez, VI, 1998, 247-264. Über Endovellicus, A. Guerra, Th. G. Schattner, C. Fabiâo-Rui Almeida: Sâo Miguel da Mota Alandroal/ Portugal, 2002. Bericht über die Ausgrabungen im Heiligtum des Endovellicus, MM, 46, 2005, 184-234.

35. Si qui viri ac mulieres divinatores, quos dicunt esse caragios atque sorticularios in quiuscumque domo gothi, romani, syri, graeci, vel iudaei fuerint invent aut quis ausus fuerit a modo in eorum vana carmina interrogare et non publico hoc voluerit....Illi vero qui tali iniquitate repleti sunt et sortes et divinationes faciunt et populum praevaricando seducunt, ubi inventi fuerint seu liberi seu servi vel ancillae sint, gravissime publice fustigentur et venundentur, et pretia, ipsorum pauperibus erogentur.

36. R. Sanz Serrano: Paganos, adivinos y magos, 70ff; A. A. Barb: «The survival of magic arts», in The Conflict between Paganism and Christinity in the fourth Century, A. Momigliano (ed), Oxford, 1963. 
Letzten Endes betrachtete der spätantike, christliche Staat den Heiden als einen fehlgeleiteten Christen und die heidnischen Priester als Magier und Wahrsager, weil die wirklichen Priester nur Christen sein konnten. Diese Transformation des Paganismus ist das Resultat der auf der Iberischen Halbinsel durchgeführten Verfolgungen. Entscheidend war aber auch, wie sich die christliche Lehre im Umfeld des Paganismus herausgebildet hatte. 